\section{Treatment of orthostatic hypotension with dihydroergotamine}

Treatment of patients with autonomic insufficiency and postural hypotension due to deficient circulatory reflexes has been unsatisfactory. Dihydroergotamine (DHE) causes selective constriction of capacitance vessels. We have investigated whether this property, by limiting venous pooling in the upright posture, ${ }^{2}$ is beneficial in postural hypotension.

\section{Methods and results}

Six patients with autonomic insufficiency and severe postural hypotension (three men, three women; mean age 67 years, range $50-80$ ) were studied. Standing systolic blood pressure while on no treatment averaged $66.7 \mathrm{~mm}$ $\mathrm{Hg}$. Fludrocortisone $0.3 \mathrm{mg} /$ day had been tried in all with little symptomatic benefit. The autonomic insufficiency was of the peripheral pattern in four patients and central in two. Autonomic insufficiency was confirmed by absent or subnormal reflex rise in peripheral vascular resistance with head-up tilting and by an absent Valsalva constrictor response. All patients were on a $160-\mathrm{mmol}$ sodium diet and had taken no drugs for four weeks before entry to the study. The effect of intravenously administered DHE on the haemodynamic response to tilting was assessed in six patients. After arterial pressure, cardiac index (by dye dilution), total peripheral resistance, and central blood volume (CBV) had been measured the patients were tilted head-up to an angle of $15^{\circ}$. In five patients this was well tolerated and the angle was increased to $30^{\circ}$. Measurements were repeated at the highest angle of tilt. DHE $10 \mu \mathrm{g} / \mathrm{kg}$ was then administered intravenously over five minutes. Supine haemodynamic measurements were repeated 10 minutes later and again after tilting.

Blood pressure increased after intravenous DHE and fell less during tilting $(P<0.01)$. Systolic pressure fell from $130+9.3 \mathrm{~mm} \mathrm{Hg}$ to $98+13$ $\mathrm{mm} \mathrm{Hg}$ before DHE, but after DHE supine systolic pressure was $171+2.9$ $\mathrm{mm} \mathrm{Hg}$ and fell to $160 \pm 8.7 \mathrm{~mm} \mathrm{Hg}$. Blood pressure was higher after DHE mainly because there was less venous pooling during tilting, with a smaller fall in CBV. Supine CBV was $1009+81 \cdot 3 \mathrm{ml} / \mathrm{m}^{2}$ before and $1114+71 \cdot 4$ $\mathrm{ml} / \mathrm{m}^{2}$ after DHE. During tilt CBV was $761 \pm 78 \cdot 2 \mathrm{ml} / \mathrm{m}^{2}$ before and $981 \pm 81 \cdot 3$ $\mathrm{ml} / \mathrm{m}^{2}$ after DHE. Changes in the cardiac index passively reflected the changes in CBV. The cardiac index during tilting was $2 \cdot 34 \pm 0.41 / \mathrm{min} / \mathrm{m}^{2}$ before and $3.29 \pm 0.31 / \mathrm{min} / \mathrm{m}^{2}$ after DHE $(P<0.01)$. Supine peripheral vascular resistance rose slightly, by $4 \mathrm{~mm} \mathrm{Hg} / 1 / \mathrm{min} / \mathrm{m}^{2}$ after DHE, suggesting some effect on resistance vessels.

The efficacy of oral DHE was then assessed in four patients. Blood pressure was measured weekly using a random zero sphygmomanometer (Gelman-Hawksley Instruments). Pressures were measured after five minutes each lying and standing. Drugs were administered single blind. Patients started on DHE $5 \mathrm{mg} /$ day in two equal doses. The dose was then increased by $5 \mathrm{mg}$ every two weeks up to $40 \mathrm{mg}$ daily. Fludrocortisone $0.3 \mathrm{mg}$ daily was then added and the combination continued for four weeks. Finally DHE was stopped (replaced by an identical placebo) and fludrocortisone alone continued until postural symptoms reappeared. The effects of oral DHE at the various doses are shown in the figure. In one patient a dose of DHE of $10 \mathrm{mg}$ daily was adequate to maintain standing blood pressure at 150/89 $\mathrm{mm} \mathrm{Hg}$ and relieve symptoms. The remaining three patients required 30 $\mathrm{mg} /$ day to raise standing blood pressure from an average of $80 / 51 \mathrm{~mm} \mathrm{H}$ to $107 / 67 \mathrm{~mm} \mathrm{Hg}(\mathrm{P}<0.001)$ with an additional rise of $28 \mathrm{~mm} \mathrm{Hg}$ when fludrocortisone was added. On withdrawal of DHE little change occurred in

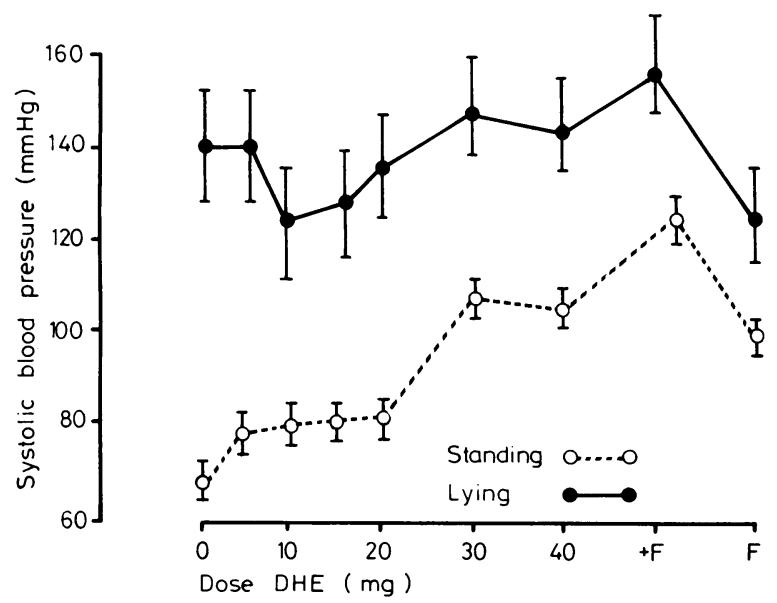

Effect of oral administration of dihydroergotamine alone, DHE plus fludrocortisone (F), and fludrocortisone alone on blood pressure. Results are means $\pm \mathrm{SE}$ of mean in four patients. blood pressure over the first two weeks. By four weeks, however, symptomatic postural hypotension had recurred. No side effects occurred during treatment with DHE alone.

\section{Comment}

Our study confirms the finding ${ }^{3}$ that intravenously administered DHE acutely improves postural hypotension in patients with autonomic insufficiency and shows that benefit results from long-term oral administration. The haemodynamic changes suggested that DHE constricts capacitance vessels so that less pooling occurs in veins on standing. ${ }^{2}$ The venoconstrictor effect of DHE seems to be due mainly to $\alpha$-adrenoceptor stimulation. ${ }^{4}$ Although DHE has constrictor and dilator effects the former would be expected to be exaggerated in the denervated vessels of these patients. ${ }^{5}$

The lack of effect of oral doses of DHE below $20 \mathrm{mg} /$ day in three patients contrasted with their response to acute intravenous DHE. The subsequent effect when the dose was increased beyond $20 \mathrm{mg}$ suggests that low bioavailability of DHE was a problem at the lower doses. In other studies doses of 2 to $10 \mathrm{mg} /$ day $^{3}$ have been used and previous "failures" may well have been due to inadequate dosage. Addition of fludrocortisone to DHE in all patients resulted in further improvement in standing blood pressure, suggesting that the two drugs have additive effects.

1 Sturmer, E, Cardiology, 1976, 61 suppl No 1, p 290.

2 Nordenfeldt, I, and Mellander, S, Acta Medica Scandinavica, 1972, 191, 115.

${ }^{3}$ Bevegard, S, Castenfors, J, and Lindblad, L-E, Acta Medica Scandinavica, 1974, 196, 473.

${ }^{4}$ Aellig, W H, European fournal of Clinical Pharmacology, 1974, 7, 137.

${ }^{5}$ Mellander, S, and Nordenfeldt, I, Clinical Science, 1970, 39, 183.

(Accepted 23 May 1979)

Clinical Research Unit, Alfred Hospital and Baker Medical Research

Institute, Commercial Road, Prahran 3181, Victoria, Australia

GARRY JENNINGS, FRACP, staff physician

MURRAY ESLER, FRACP, PHD, assistant physician

ROMAYNE HOLMES, MB, BS, registrar

\section{Interaction between chloramphenicol and paracetamol}

While studying hepatic microsomal glucuronidation in children with kwashiorkor, we observed that when chloramphenicol and paracetamol were given together the half life of chloramphenicol was prolonged from the normal 2-3 hours ${ }^{1}$ to $18-24$ hours. We then decided to study this interaction in adults receiving chloramphenicol in an intensive care unit.

\section{Patients, methods, and results}

Six patients in the intensive care unit, all of whom had an infection and had received broad-spectrum antibiotics, were given chloramphenicol, $1 \mathrm{~g}$ intravenously. Blood samples were taken after $\frac{1}{2}, 1,1 \frac{1}{2}$, and 2 hours and the chloramphenicol concentration measured. ${ }^{1}$ The patients were then given paracetamol, $100 \mathrm{mg}$ intravenously, and further blood samples were taken at $3,4,5$, and 6 hours after the chloramphenicol had been given. Thereafter the patients received a full course of chloramphenicol.

The results (figure) show that before paracetamol was given the half life of chloramphenicol was $3 \cdot 25$ hours. After paracetamol was given, however, the elimination of chloramphenicol was reduced, and the half life of chloramphenicol increased to 15 hours.

\section{Comment}

Chloramphenicol usage and toxicity have been reviewed. ${ }^{2}$ The inhibition of phenytoin, tolbutamide, chlorpropamide, and dicoumarol metabolism by chloramphenicol is well known. ${ }^{3}$ We suggest that chloramphenicol elimination is appreciably decreased by simultaneously giving paracetamol, probably because of competitive metabolism. This is important because, at least in underdeveloped societies, chloramphenicol is widely used, being cheap and readily available and the usual treatment for typhoid. It is often given with an 


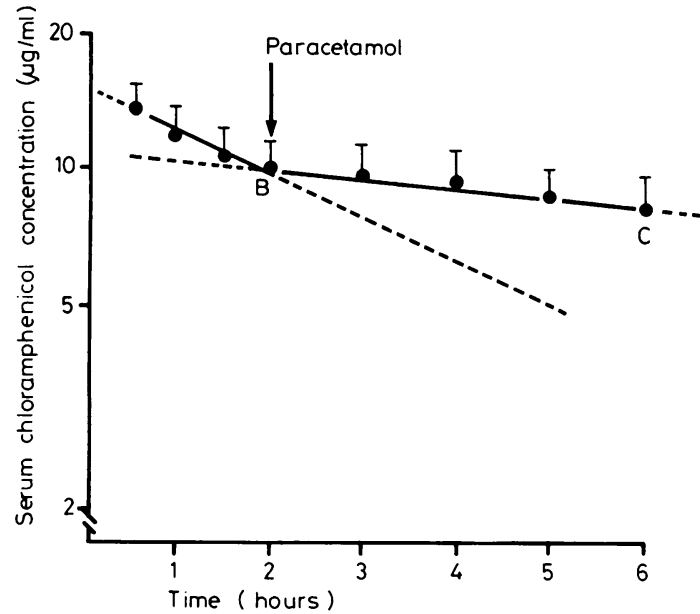

Effect of paracetamol administration on elimination of chloramphenicol. Before paracetamol administration (A-B) chloramphenicol half life was 3.25 hours, whereas after chloramphenicol administration (B-C) the half life increased to 15 hours.

antipyretic, such as paracetamol, to treat typhoid. Ideally, chloramphenicol and paracetamol should not be prescribed together, but if they are either the dose of chloramphenicol should be reduced or serum chloramphenicol concentrations should be measured.

${ }^{1}$ Glasko, A J, et al, fournal of Pharmacology and Experimental Therapeutics, $1949,96,445$.

${ }^{2}$ Haile, C A, Southern Medical fournal, 1977, 70, 479.

${ }^{3}$ Christensen, L K, and Skovsted, L, Lancet, 1969, 2, 1297.

(Accepted 1 fune 1979)

Intensive Care Unit and Metabolic and Nutrition Research Unit,

Baragwanath Hospital, Johannesburg, South Africa

N BUCHANAN, FCP, PHD, consultant paediatrician

G P MOODLEY, DMT, senior laboratory technician

\section{Chuei-Fong-Tou-Geu-Wan in rheumatoid arthritis}

We describe a patient with rheumatoid arthritis (RA) who showed astonishing improvement while taking a Chinese "herbal" medicine.

\section{Case report}

A 44-year-old Dutch housewife, temporarily living in Birmingham, presented in November 1977 with a seven-month history of increasing morning stiffness and pain in feet, knees, and hands. There had been no prodromal illness. She had a symmetrical polyarthritis of the small joints and effusions in both knees. Investigations showed: haemoglobin concentration $12.6 \mathrm{~g} / \mathrm{dl}$; erythrocyte sedimentation rate (ESR) $18 \mathrm{~mm}$ in first hour; serum C-reactive protein (CRP) concentration $9 \mathrm{mg} / 1$; rheumatoid factor and antinuclear factor absent; viral complement fixation tests negative; no diagnostic features in radiographs of chest and affected joints.

She was treated for two months with non-steroidal anti-inflammatory drugs but felt no better; ESR and acute-phase protein concentrations increased (figure). In January 1978 ICI 55 897, a new drug under trial for RA, ${ }^{1}$ was begun but in March she was still deteriorating. Two weeks later she began Chuei-Fong-Tou-Geu-Wan 12 pills daily and within two days all pain had gone; the improvement was confirmed by objective criteria (figure). She remained well but over the ensuing three months noticed that reducing the dose resulted in a return of pain; there was an associated increase in serum CRP. Improvement was regained by returning to the higher dose.

\section{Comment}

After taking non-steroidal anti-inflammatory drugs for two months this patient needed more potent treatment. Continuing deterioration
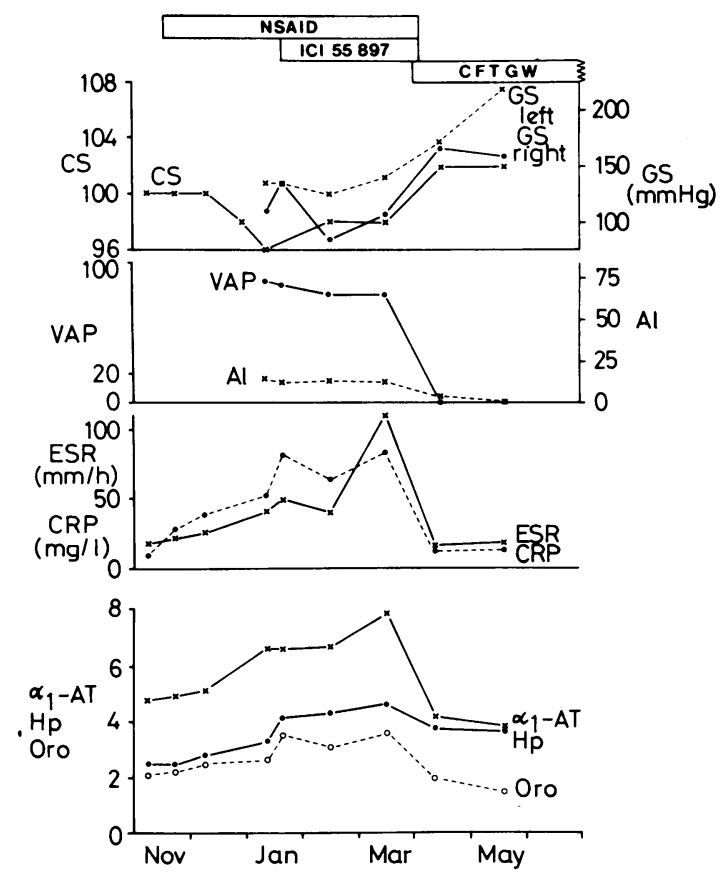

Subjective clinical score (CS), grip strength (GS), visualanalogue pain scale (VAP), articular index (AI), erythrocyte sedimentation rate (ESR), and serum concentrations of Creactive protein $(\mathrm{CRP}), \alpha_{1}$-antitrypsin $\left(\alpha_{1}-\mathrm{AT}\right)$, haptoglobin (Hp), and orosomucoid (Oro) during treatment with nonsteroidal anti-inflammatory drugs (NSAID) and ICI 55897 and after beginning Chuei-Fong-Tou-Geu-Wan (CFTGW).

while taking ICI 55897 , although this drug has a slow action, might soon have necessitated a further change. Our choice was pre-empted because she took Chuei-Fong-Tou-Geu-Wan at the suggestion of friends in Holland, where the drug was receiving attention as a highly effective remedy for arthritis. According to the manufacturers the pills contained 23 herbal substances-the principal constituent being Apis chinensis - and "repel poisonous factors and helcosis." Her improvement was so impressive that we agreed to her continuing. The effect of the drug on her symptoms and acute-phase serum protein concentrations, however, suggested that it contained corticosteroids or synthetic analogues; no other drug has so abrupt and profound an effect. ${ }^{2}$ Furthermore, after three months' treatment she began to look Cushingoid, which together with a low plasma cortisol concentration $(7 \mathrm{nmol} / \mathrm{l} ; 0.25 \mathrm{~kg} / 100 \mathrm{ml})$ suggested exogenous corticosteroid administration. By September public interest in Holland had led to analysis of the pills, the results from the Government Institute for Medicines being published in the press ${ }^{3}$ under the headline "Hongkong-pil Chinese roulette." The tablets contained dexamethasone and indomethacin.

The composition of Chuei-Fong-Tou-Geu-Wan, which, so far as we can tell, means "chase-the-wind-through-the-bones tablets," apparently varies. In the San Francisco Bay area it was implicated in four cases of agranulocytosis, one of which was fatal ${ }^{4}$; analysis yielded aminopyrine and phenylbutazone. Interestingly, no cases of agranulocytosis were seen in Chinese patients, suggesting that the addition of non-steroidal anti-inflammatory drugs was particularly for the Western market. We finally suggested that our patient should stop the pills, and she is now well, taking prednisolone $3.75 \mathrm{mg}$ daily.

${ }^{1}$ McConkey, B, et al, Annals of the Rheumatic Diseases. In press.

2 McConkey, B, et al, Quarterly fournal of Medicine, 1973, 42, 785.

${ }^{3}$ Ad Zaterdag, 30 September 1978.

${ }^{4}$ Curt, A R, and Mervyn, A S, fournal of the American Medical Association, 1975, 231, 352.

(Accepted 18 fune 1979)

Dudley Road Hospital, Birmingham B18 7QH

P J G FORSTER, MRCP, research registrar

M CALVERLEY, SRN, clinical metrologist

$S$ HUBBALL, BSC, AIMLS, technician

B MCCONKEY, DM, FRCP, consultant physician 